\title{
Black Holes Often Saturate Entanglement Entropy the Fastest
}

\author{
Márk Mezei®* \\ Simons Center for Geometry and Physics, SUNY, Stony Brook, New York 11794, USA \\ Wilke van der Schee ${ }^{\dagger}$ \\ Theoretical Physics Department, CERN, CH-1211 Genève 23, Switzerland
}

(Received 22 January 2020; revised manuscript received 13 April 2020; accepted 4 May 2020; published 20 May 2020)

\begin{abstract}
There is a simple bound on how fast the entanglement entropy of a subregion of a many-body quantum system can saturate in a quench: $t_{\mathrm{sat}} \geq R / v_{B}$, where $t_{\mathrm{sat}}$ is the saturation time, $R$ the radius of the largest inscribed sphere, and $v_{B}$ the butterfly velocity characterizing operator growth. By combining analytic and numerical approaches, we show that in systems with a holographic dual, the saturation time is equal to this lower bound for a variety of differently shaped entangling surfaces, implying that the dual black holes saturate the entanglement entropy as fast as possible. This finding adds to the growing list of tasks that black holes are the fastest at. We furthermore analyze the complete time evolution of entanglement entropy for large regions with a variety of shapes, yielding more detailed information about the process of thermalization in these systems.
\end{abstract}

DOI: 10.1103/PhysRevLett.124.201601

Introduction.-The time evolution of entanglement entropy (EE) is an interesting detailed probe of thermalizing many-body systems [1-7]. By causality, the EE $S[A(t)]$ of a subregion $A$ can never saturate to its thermal equilibrium value faster than a time $t_{\mathrm{sat}} \geq t_{\mathrm{LC}}$, with $t_{\mathrm{LC}}$ the time to the tip of the past light cone of region $A$. Geometrically, $t_{\mathrm{LC}}=R / c$ with $R$ the radius of the largest inscribed sphere in $A$ and $c$ the speed of light [8]. Using insight from chaotic operator growth $[9,10]$, the bound on the saturation time can be improved to $t_{\text {sat }} \geq R / v_{B}$ [11], with $v_{B}$ the butterfly velocity characterizing the spreading footprint of operators. This improved bound is the main interest of this Letter.

Solving for the time evolution of EE is a very challenging problem. Results are available in special solvable examples: two-dimensional conformal field theories [12,13], free theories [14,15], random quantum circuits [16-18], and holographic gauge theories [3-5,11,19-23]. Recently, much progress has been made in understanding the process of $\mathrm{EE}$ growth in generic chaotic systems in a "hydrodynamic" limit $R, t \gg t_{\text {loc }}$ (with $t / R$ fixed), where $t_{\text {loc }}$ is the local thermalization timescale. For any region, the entropy starts to grow linearly according to the universal law $[3-6,12,13]$

$$
S[A(t)]=s_{\mathrm{th}} v_{E} \operatorname{area}(\partial A) t+\cdots,
$$

Published by the American Physical Society under the terms of the Creative Commons Attribution 4.0 International license. Further distribution of this work must maintain attribution to the author(s) and the published article's title, journal citation, and DOI. Funded by SCOAP ${ }^{3}$. where $S[A(t)]$ is the vacuum subtracted EE of region $A(t), s_{\text {th }}$ is the thermal entropy density, and the entanglement velocity $v_{E}$ is defined by this equation. In the hydrodynamic limit, the growth of EE as a function of the time can be described by an effective membrane theory $[17,24,25]$, which states that (the leading piece of) $S[A(t)]$ after a quench at $t=0$ in any chaotic system is computed by a membrane minimizing the functional

$S[A(t)]=s_{\text {th }} \int_{0}^{t} d \operatorname{area} \frac{\mathcal{E}(v)}{\sqrt{1-v^{2}}}, \quad v \equiv \frac{\left(n^{\mu} \hat{t}_{\mu}\right)}{\sqrt{1+\left(n^{\mu} \hat{t}_{\mu}\right)^{2}}}$,

where the timelike membrane stretches between two planes in the $d$-dimensional Minkowski spacetime that the system lives in: It is anchored at $t$ on the upper face on the entangling surface $\partial A(t)$ and ends perpendicularly (on an arbitrary shape) on the lower plane at $t=0$. Here, $n^{\mu}$ is its local unit normal $\left(n^{2}=1\right)$, and $\hat{t}^{\mu}=(1, \mathbf{0})$ is the timelike unit vector, and the "velocity" $v$ is determined by their angle; see Fig. 1. Since the membrane is timelike it follows that $|v| \leq 1$. $\mathcal{E}(v)$ is the Lagrangian referred to as the "angle-dependent membrane tension" that we elaborate on below.

The functional was originally presented based on analytic results on random quantum circuits [17,24], where the membrane is to be thought of as a coarse-grained cut through the tensor network representing the evolving wave function. In holographic gauge theories, the $\mathrm{EE}$ is given by the area of an extremal surface in the dual spacetime that ends on $\partial A(t)$ on the spacetime boundary (that hosts the dual gauge theory) [19-21]. In the hydrodynamic limit, it was shown in Ref. [25] that the holographic extra dimension can be integrated out, giving the membrane 


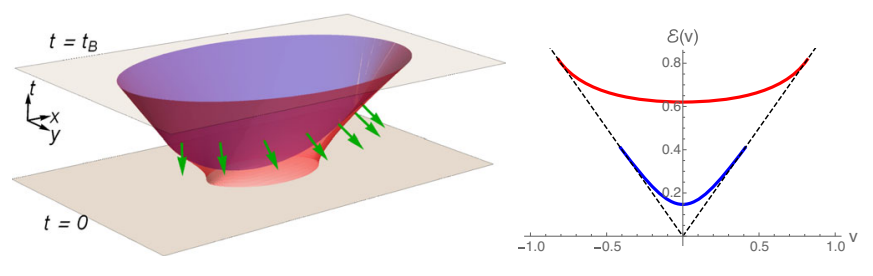

FIG. 1. (Left) A membrane (red) anchored on an ellipse $A$ shown together with normal vectors (green), the timelike unit vector, and the two planes in Minkowski space. The $v_{B}$ light sheet over the same ellipse (a membrane with $v=v_{B}$ ) is drawn in blue and just touches the plane at $t=0$. (Right) The membrane tension function of Eq. (5) (red) and a charged quench for comparison (blue). The dashed black line is at $45^{\circ}$. They touch at $v=v_{B}$.

theory (2). In the holographic case, the membrane is given by the projection of the extremal surface computing EE in the gravitational description onto the spacetime boundary along constant infalling time.

These complementary ways of thinking about the membrane add to the intriguing connections between cuts through tensor networks and holographic extremal surfaces pioneered in Refs. [3,10,26-29]. The membrane theory can be generalized in many directions, which demonstrates its robustness [30-35]. Since it applies equally to very different systems-holographic gauge theories and random quantum circuits-and since it has all the ingredients to be adapted to all chaotic systems, we take the view that it is a universal effective theory of EE dynamics in much the same way as hydrodynamics describes the evolution of conserved charge densities. The derivation of the effective membrane theory for general chaotic systems however remains an open challenge.

Specifying $\mathcal{E}(v)$.- - Like the equation of state or transport coefficients in hydrodynamics, the angle-dependent membrane tension $\mathcal{E}(v)$ depends on the theory and conserved charges, but not on other details of the state whose EE dynamics we are studying. $\mathcal{E}(v)$ obeys the following general constraints: It is an even function of $v$, monotonically increasing for $v>0$, convex, interpolates between $\mathcal{E}(0)=v_{E}$ and $\mathcal{E}\left(v_{B}\right)=v_{B}$, and is tangent to the $45^{\circ}$ line at $v_{B}, \mathcal{E}^{\prime}\left(v_{B}\right)=1$.

In random quantum circuits, $\mathcal{E}(v)$ depends on the structure of the circuit: In one simple $1+1 \mathrm{D}$ example with a large on-site Hilbert space, $\mathcal{E}(v)=\frac{1}{2}\left(1+v^{2}\right)$ [17], but no results are known for higher dimensions. In holography, $\mathcal{E}(v)$ repackages the dual spacetime geometry. A field theory quench is dual to a spacetime in which a black hole forms from collapse. The equilibrium black hole does not depend on the details of the quench, only the conserved charges, and this is the region of spacetime that determines $\mathcal{E}(v)$. Its metric can be written as

$$
\begin{aligned}
d s^{2} & =\frac{1}{z^{2}}\left[-a(z) d t^{2}-2 d t d z+d \vec{x}^{2}\right], \\
a(z) & =1-M z^{d}+Q^{2} z^{2(d-1)},
\end{aligned}
$$

where $(t, \vec{x})$ are the field theory coordinates and $z$ is the holographic extra dimension, and as an example, we chose a family of charged black holes with $M$ the mass and $Q$ the charge, which map to the energy and charge density of the state in the field theory. Then, $\mathcal{E}(v)$ is given parametrically by the formula

$$
\{v(z), \mathcal{E}[v(z)]\}=\left\{\sqrt{a(z)-\frac{z a^{\prime}(z)}{2(d-1)}}, \sqrt{\frac{-a^{\prime}(z)}{2(d-1) z^{2 d-3}}}\right\},
$$

where $z \in\left[0, z_{*}\right]$ and $z_{*}$ is the value for which $v\left(z_{*}\right)=0$ [25]. In this Letter, we will study in detail the case $d=4$, $Q=0$, describing charge neutral quenches in 4D holographic field theories, for which Eq. (4) evaluates to

$\mathcal{E}(v)=\frac{v_{E}}{\left(1-v^{2}\right)^{1 / 4}}, \quad v_{E}=\frac{\sqrt{2}}{3^{3 / 4}}, \quad v_{B}=\sqrt{\frac{2}{3}} ;$

see Fig. 1 (right) [36]. On occasion, we will present results for charged black holes, whose dynamics is expected to be slower due to the decrease of the size of the available Hilbert space [37]. Our methods and (most of our) results straightforwardly extend to other $\mathcal{E}(v)$ 's and hence conjecturally to any chaotic system. While currently the only higher-dimensional examples for $\mathcal{E}(v)$ come from holography, once new analytic or numerical $\mathcal{E}(v)$ functions become known for nonholographic systems, it will be very interesting to revisit our results for them.

In the following, we compute the time evolution of $\mathrm{EE}$ in the membrane theory (2) with $\mathcal{E}(v)$ given in Eq. (5) (and some charged generalizations) for a variety of entangling regions, which compute the $\mathrm{EE}$ in holographic field theories in the hydrodynamic regime. Besides determining the full time evolution, we analyze the saturation time in detail.

Analytic results.-We briefly review the analytic solution of the membrane theory for symmetric shapes. For $A$ a strip the membrane is a straight plane stretching between the $t=0$ and $t$ time slices. Evaluating Eq. (2) for this membrane gives linear growth with slope $s_{\text {th }} v_{E}$ area $[\partial A]$ until saturation, consistent with Eq. (1). The saturation time for a strip of width $2 R$ is hence $t_{\text {sat }}=R / v_{E} \equiv t_{E}$. For spherical $A$, the action reduces to

$$
S[A(t)]=4 \pi s_{\text {th }} \int_{0}^{t} d s \rho^{2}(s) \mathcal{E}[\dot{\rho}(s)],
$$

where $s$ is the time coordinate $0 \leq s \leq t$, and we describe the membrane with a radial coordinate $\rho(s)$ [hence, $v=\dot{\rho}(s)$ ]. Minimizing Eq. (6) is a one-dimensional classical mechanics problem, and can be straightforwardly solved using energy conservation. $S[A(t)]$ is shown in Fig. 4 (as the $r=1$ curve) for the membrane tension 

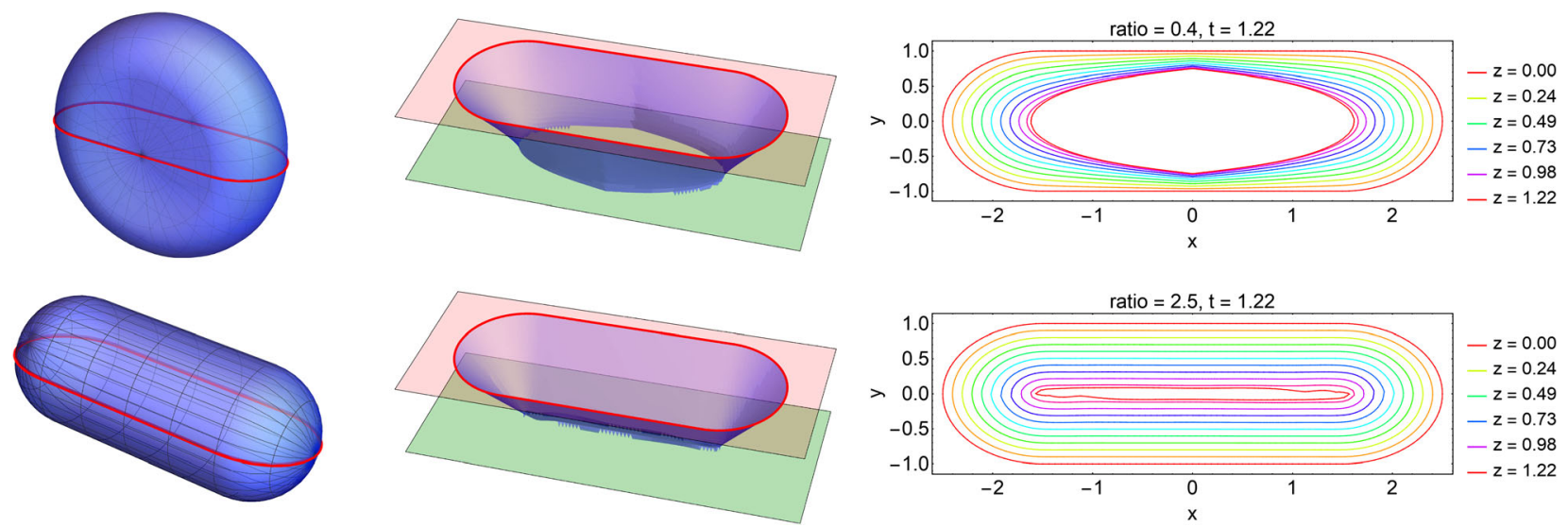

FIG. 2. Detailed illustration of the relevant membranes for rotated stadia with short-to-long ratio of 2.5 (top) and 0.4 (bottom) at $t=1.22$, just before the butterfly time $t_{B}=1 / v_{B} \approx 1.2247$. The largest inscribed sphere has radius $R=1$. Curiously, even for this nontrivial shape, the EE saturates as fast as possible for the 2.5 case.

corresponding to the neutral black hole (5). The curve ends at $t_{B}$ : The corresponding membrane has $v(s)=v_{B}$ and hence is a $v_{B}$ light sheet. The problem for a cylinder subregion is solved by replacing $4 \pi \rho^{2}(s) \rightarrow 2 \pi L \rho(s)$ in Eq. (6). Remarkably, the cylinder also saturates at $t_{\text {sat }}=t_{B}$; see Fig. 4 (as the $r=\infty$ curve). Below we will find that shapes that interpolate between the sphere and cylinder saturate EE at $t_{B}$, while those that interpolate between the sphere and the strip have $t_{B} \leq t_{\text {sat }} \leq t_{E}$.

We have also analyzed charged quenches whose EE dynamics is governed by $\mathcal{E}(v)$ computed from Eq. (4). We find three regimes as a function of $q \equiv Q / M^{3 / 4}$ : For $0 \leq q \leq 0.38$, both the sphere and the cylinder (and we expect that all the shapes that interpolate between them) have $t_{\mathrm{sat}}=t_{B}$; for $0.38 \leq q \leq 0.61$, the sphere has $t_{\mathrm{sat}}=t_{B}$, while the cylinder has $t_{\text {sat }}>t_{B}$ (and hence, among the shapes that interpolate between them, there should be an open set with $t_{\text {sat }}=t_{B}$ ), while for $0.61 \leq q$, all shapes have $t_{\text {sat }}>t_{B}$. We present a detailed discussion of $S[A(t)]$ for a spherical region from this last regime of $q$, for $q=0.62$ in the Supplemental Material [38].

For more general shapes, the minimal membrane will be solved numerically in the next section, but it is possible to obtain rather constraining analytic upper bounds on the entropy [11]. In the framework of the membrane theory (2), this bound corresponds to considering a variational surface consisting of two parts joined at $t=t^{\prime}$ : a "light sheet" part of slope $v_{B}$ and a vertical tube with $\mathcal{E}(v=0)=v_{E}$. We get a tight upper bound by minimizing in $t^{\prime}$ the total membrane action

$$
\begin{aligned}
S_{\max }[A(t)]= & s_{\text {th }} \min _{0 \leq t^{\prime} \leq \min \left(t, t_{B}\right)}\left[\left\{\operatorname{vol}(A)-\operatorname{vol}\left(A^{\prime}\right)\right\}\right. \\
& \left.+v_{E} \operatorname{area}\left(A^{\prime}\right) t^{\prime}\right],
\end{aligned}
$$

where $A^{\prime}\left(t^{\prime}\right)$ is the past butterfly domain of dependence of $A(t)$ at time $t^{\prime}$, i.e., the set of points in region $A$ that are farther from $\partial A$ than $v_{B}\left(t-t^{\prime}\right)$. We show $S_{\max }[A(t)]$ on
Fig. 3 together with the numerical results for $S[A(t)]$. The bounds are very close to the actual results (see also Ref. [11] for similar results for spherical regions).

Numerical results. - In general, the minimization of the action (2) cannot be solved analytically, but it is possible to start with some initial surface and gradually relax this surface to a (local) minimal solution. For this, we used SURFACE EVOLVER [39], which uses a triangulation of the surface to minimize some energy functional. We implemented this in 3D for ellipses and stadia (consisting of two half-circles connected by lines) having a ratio $r$ between the long and short axes. In 4D, we kept one rotational symmetry by rotating these surface about the long axis [Fig. 2 (top)] or short axis [Fig. 2 (bottom)]. The numerical implementation is included in the Supplemental Material [38].

We now briefly describe some general features of $S[A(t)]$ and the membranes computing it shown in Figs. 2-4. In the early time regime captured by Eq. (1), the membrane has $v \approx 0$; i.e., it is a tube stretching between the upper and lower ends of the spacetime slab. After this early time regime, $S[A(t)]$ starts to curve, and smoothly saturates to the thermal value $S[A(t)]=s_{\mathrm{th}} \operatorname{vol}(A)$ at some saturation time $t_{\text {sat }}$. At intermediate times, the membrane generically forms cusps, most visible on Fig. 2. Since the action depends on $v$ but not its derivatives, the jump of $v$ at the cusp does not cause a divergence in the action.

Saturation time.-A remarkable feature of Figs. 3 (left) and 4 (top) is that EE saturates at the butterfly time $t_{B}=R / v_{B}$, where $R$ is the radius of the largest inscribed sphere inside the entangling surface. We found the same feature in prolate ellipsoids; the numerical results are included in the Supplemental Material [38]. Independent of the validity of the membrane theory, Ref. [11] gave an argument that the bound $t_{\text {sat }} \geq t_{B}$ holds in any many-body system. The saturation of this bound is our most important result and allows us to conclude that neutral (and 

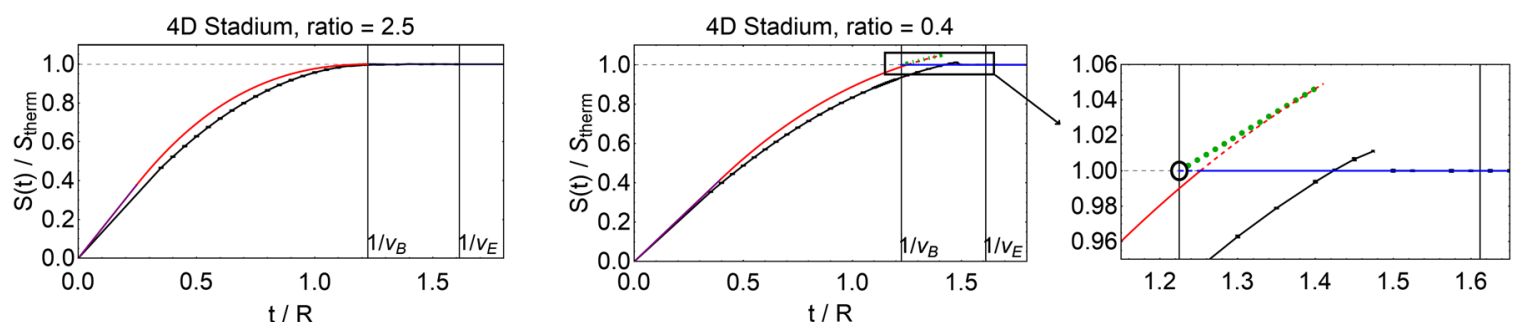

FIG. 3. Time evolution of the EE for stadia of Fig. 2. The time evolution obeys the analytic bound of Eq. (7) plotted by a red solid line when they are minimal and dashed where they become nonminimal. The $v_{B}$ light sheet at $t_{B}$ is indicated by a black circle, which lies at the end of a dashed green line representing a disfavored branch of variational membranes.

moderately charged) black holes often saturate entanglement entropy the fastest, where "often" indicates a large class of shapes.

We now explain the often qualifier of this statement. It is not true that for all shapes the saturation time is as fast as possible. For the analytically solvable case of the strip, we get $t_{\mathrm{sat}}=t_{E}>t_{B}$. (Recall that $t_{E}=R / v_{E}$.) For the squashed stadium of Figs. 2 and 3 and also other stadia on Fig. 4, as well as oblate ellipsoids (see the Supplemental Material [38]), we find $t_{\mathrm{sat}}>t_{B}$. In the case of the stadia, this can be proven analytically: We show that even $S_{\max }[A(t)]$ saturates later than $t_{B}$, and hence, so does $S[A(t)]$ (see Fig. 3 for an example). In the inset of Fig. 4, we show the analytic lower bound on the saturation time that interpolates between $t_{B}$ and $t_{E}$ together with the numerical results. That for $r \rightarrow 0$ we get $t_{\mathrm{sat}}=t_{E}$ is expected, since in this limit the squashed stadium becomes a strip. We note that in the membrane theory, one can prove a simple upper bound on the saturation time $t_{\mathrm{sat}} \leq t_{E}$ [25], so the family of shapes that we consider realize all possible saturation times.

It would be bold to conclude that $t_{\mathrm{sat}}=t_{B}$ for a large family of shapes based solely on numerical data. We now make our case stronger by presenting a semianalytic argument to this effect. Interestingly, we can establish analytically that at $t=t_{B}$, the $v_{B}$ light sheet over the entangling surface is a (locally) minimal surface [25]. [This is also a surface that is contained in the set of variational surfaces in Eq. (7) for $t^{\prime}=0$.] In some cases, it is also the global minimum, and we have $t_{\text {sat }}=t_{B}$; see Fig. 2. In other cases, as shown on Fig. 3, lower left corner, we see that there is a variational surface with $t^{\prime}>0$ that gives lower entropy (red line at $t=t_{B}$ ), even though the numerical membrane gives even lower entropy (black line). At some time $t_{\text {sat,var }}>t_{B}$, the variational surface gives a larger entropy than the thermal value, after which we continue the (disfavored) variational estimate as dashed line. It is this transition that gives a lower bound on the actual saturation time, as shown in the inset of Fig. 4. At an even later time, it is not possible to connect the two surfaces with a regular minimal variational membrane; we indicated the smooth transition from that point to the $v_{B}$ light sheet (black circle) by a dashed green line in Fig. 3 .
Numerically, we were only able to follow the favored branch, but we believe that the disfavored branch also exists. These two branches are the only ones that give membranes that connect the two ends of the spacetime slab. On top of these, the membrane theory also allows for a horizontal membrane that gives $S[A(t)]=s_{\mathrm{th}} \operatorname{vol}(A)$. This is the horizontal line on all figures, colored blue on Fig. 3. For $t>t_{\text {sat }}$ it is the minimal membrane.

The existence of multiple branches of minimal membranes is the mechanism by which the $v_{B}$ light sheet fails to be the relevant membrane at $t=t_{B}$. Another piece of evidence for this mechanism comes from the analytic study of spherical entangling surfaces for membrane tensions $\mathcal{E}(v)$ that result from a charged quench in holographic theories, where a favored and disfavored branch was seen in Ref. [22] (for completeness, included in the Supplemental Material [38]). From understanding the
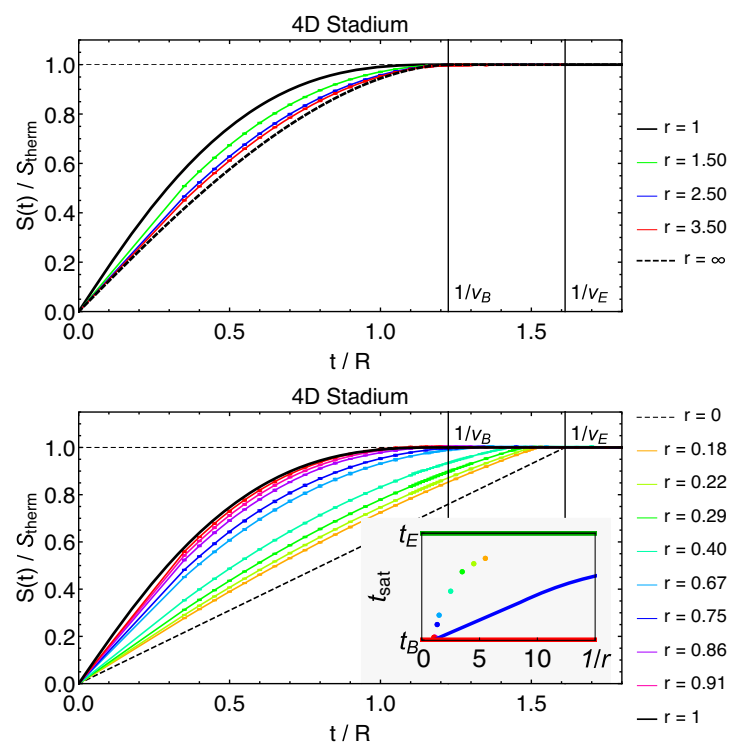

FIG. 4. See Fig. 2 for illustrations of the rotated stadia. Here we show for several shapes the EE. The analytically solvable sphere, cylinder, and strip are shown in black and correspond to ratios 1, 0 , and $\infty$, respectively. The inset shows the saturation time (colored dots) for rotated stadia, together with the analytic lower bound (solid blue) coming from Eq. (7) (see the red line in Fig. 3 for an example with $r=0.4$ ). 
mechanism for how we get $t_{\text {sat }}>t_{B}$, it follows that we only have to understand if the two branches exist or not. In the latter case, we get $t_{\mathrm{sat}}=t_{B}$. In this Letter, we decide this question numerically.

We also note that depending on which situation we are in, the slope of the curve, $\left.(d / d t) S[A(t)]\right|_{t=t_{\text {sat }}}=$ finite for the case $t_{\text {sat }}>t_{B}$, and zero when $t_{\text {sat }}=t_{B}$ (see Fig. 3).

Discussion.-In this Letter, we studied the EE $S[A(t)]$ in the hydrodynamic regime, of large regions at late times, in the membrane effective theory proposed to capture the dynamics of entanglement in all chaotic systems. By focusing on the membrane tension function $\mathcal{E}(v)$ given in Eq. (5), we specialized to the case of 4D holographic gauge theories and neutral quenches, but all our methods generalize to any theory, once $\mathcal{E}(v)$ is provided as an input. We also derived some results for charged quenches in the same theories.

One important problem we leave for the future is how to determine whether $t_{\mathrm{sat}}=t_{B}$ or larger analytically without having to solve for the minimal membrane numerically. This may be possible by perturbing around the $v_{B}$ light sheet membrane to decide if it is on the dominant or disfavored branch, as in Figs. 2 or 3, respectively.

While in the case of holography, we have a way of computing not just the leading piece in the hydrodynamic limit, but the exact entropy (using the holographic surface extremization prescription [19-21]). Even in this context there are many advantages of using the membrane theory: The simplified description allows for the identification of features that survive the hydrodynamic limit, enables the understanding of near saturation behavior, and the numerical solution of the relatively simple-looking surface extremization problem is prohibitive in the presence of a large-scale separation $R, t \gg \beta$. In generic chaotic theories, the determination of the exact entropy is out of reach, but one may hope that in the future we will learn how to determine $\mathcal{E}(v)$ from other data characterizing the theory. One hint that this may be possible is that we can determine the special point $\mathcal{E}\left(v_{B}\right)=v_{B}$ by studying out-of-time-order correlators. Another way to exploit the power of the membrane theory is to determine $\mathcal{E}(v)$ from determining $S[A(t)]$, e.g., for a sphere in numerics [see Ref. [24] for a related numerical determination of $\mathcal{E}(v)]$ or in the future in experiments (see Ref. [7] for early experimental work), which then yields infinitely many new predictions for other shapes.

In the future, we can use the numerical methods developed here to study the EE in inhomogeneous quench setups, where the membrane theory is coupled to the hydrodynamic degrees of freedom [34]. One fascinating application would be to understand whether signatures of turbulent fluid flows show up in EE.

Our most interesting finding is that moderately charged black holes produce EE dynamics with saturation time $t_{\text {sat }}=t_{B}$ for elongated shapes that interpolate between a sphere and the cylinder, which is the fastest possible allowed by quantum mechanics [11]. This adds to the list of things black holes excel at: They are also fastest scramblers [9,40,41], have Planckian equilibration time $[42,43]$, and are conjectured to have the lowest sheer viscosity-to-entropy-density ratio in nature [44].

To highlight how efficient black holes are at saturating entropy, we conclude by contrasting their chaotic dynamics to those of free field theories (in Gaussian states), whose EE dynamics is expected to be modeled by the quasiparticle theory [12-15]. As shown in Ref. [14], this model gives $t_{\text {sat }}=d_{\max } / 2$, where $d_{\max }$ is the largest distance between two points of the subregion. The intuition behind this result is simple: This is the time when one member of any quasiparticle pair free streaming at the speed of light has left the subregion $A$. Since we have to associate $v_{B}=1$ with these systems, only spheres can have $t_{\text {sat }}=t_{B}$, and all elongated shapes give longer saturation times $t_{\text {sat }}>t_{B}$ with $t_{\text {sat }}=\infty$ for the cylinder.

We thank Julio Virrueta and Amos Yarom for useful discussions. M. M. thanks the KITP for its hospitality during the final stages of this work, where his research was supported in part by the National Science Foundation under Grant No. NSF PHY-1748958. W. S. is partially supported by the Netherlands Organisation for Scientific Research (NWO) under Veni grant 680-47-458.

*mmezei@scgp.stonybrook.edu

†wilke.van.der.schee@cern.ch

[1] J. H. Bardarson, F. Pollmann, and J. E. Moore, Phys. Rev. Lett. 109, 017202 (2012).

[2] M. Serbyn, Z. Papić, and D. A. Abanin, Phys. Rev. Lett. 110, 260601 (2013).

[3] T. Hartman and J. Maldacena, J. High Energy Phys. 05 (2013) 014.

[4] H. Liu and S. J. Suh, Phys. Rev. Lett. 112, 011601 (2014).

[5] H. Liu and S. J. Suh, Phys. Rev. D 89, 066012 (2014).

[6] H. Kim and D. A. Huse, Phys. Rev. Lett. 111, 127205 (2013).

[7] A. M. Kaufman, M. E. Tai, A. Lukin, M. Rispoli, R. Schittko, P. M. Preiss, and M. Greiner, Science 353, 794 (2016).

[8] T. Hartman and N. Afkhami-Jeddi, arXiv:1512.02695.

[9] S. H. Shenker and D. Stanford, J. High Energy Phys. 03 (2014) 067.

[10] D. A. Roberts, D. Stanford, and L. Susskind, J. High Energy Phys. 03 (2015) 051.

[11] M. Mezei and D. Stanford, J. High Energy Phys. 05 (2017) 065.

[12] P. Calabrese and J. L. Cardy, J. Stat. Mech. (2005) P04010.

[13] P. Calabrese and J. Cardy, J. Stat. Mech. (2007) P06008.

[14] H. Casini, H. Liu, and M. Mezei, J. High Energy Phys. 07 (2016) 077.

[15] J. S. Cotler, M. P. Hertzberg, M. Mezei, and M. T. Mueller, J. High Energy Phys. 11 (2016) 166. 
[16] A. Chandran and C. R. Laumann, Phys. Rev. B 92, 024301 (2015).

[17] A. Nahum, J. Ruhman, S. Vijay, and J. Haah, Phys. Rev. X 7, 031016 (2017).

[18] A. Nahum, J. Ruhman, and D. A. Huse, Phys. Rev. B 98, 035118 (2018).

[19] S. Ryu and T. Takayanagi, Phys. Rev. Lett. 96, 181602 (2006).

[20] S. Ryu and T. Takayanagi, J. High Energy Phys. 08 (2006) 045.

[21] V. E. Hubeny, M. Rangamani, and T. Takayanagi, J. High Energy Phys. 07 (2007) 062.

[22] M. Mezei, J. High Energy Phys. 05 (2017) 064.

[23] M. Mezei and J. Virrueta, arXiv:1909.00919.

[24] C. Jonay, D. A. Huse, and A. Nahum,arXiv:1803.00089.

[25] M. Mezei, Phys. Rev. D 98, 106025 (2018).

[26] B. Swingle, Phys. Rev. D 86, 065007 (2012).

[27] B. Swingle, arXiv:1209.3304.

[28] F. Pastawski, B. Yoshida, D. Harlow, and J. Preskill, J. High Energy Phys. 06 (2015) 149.

[29] P. Hayden, S. Nezami, X.-L. Qi, N. Thomas, M. Walter, and Z. Yang, J. High Energy Phys. 11 (2016) 009.

[30] T. Zhou and A. Nahum, Phys. Rev. B 99, 174205 (2019).

[31] N. Bao and M. Mezei, arXiv:1811.00019.

[32] J. Kudler-Flam, M. Nozaki, S. Ryu, and M. T. Tan, J. High Energy Phys. 01 (2020) 031.
[33] C. A. Agon and M. Mezei, arXiv:1910.12909.

[34] M. Mezei and J. Virrueta, J. High Energy Phys. 02 (2020) 013.

[35] T. Zhou and A. Nahum, arXiv:1912.12311.

[36] In 2D, every subregion is an interval, and the entropy grows linearly with time with the rate $v_{E}$. In 3D, even spheres do not saturate the saturation time bound [22,25]; hence, we chose to study the 4D case.

[37] S. Leichenauer, Phys. Rev. D 90, 046009 (2014).

[38] See Supplemental Material at http://link.aps.org/ supplemental/10.1103/PhysRevLett.124.201601 for an analysis of spherical regions (charged and neutral), ellipsoidal regions (neutral 3D and 4D) and more details on the numerical procedure.

[39] K. A. Brakke, Exp. Math. 1, 141 (1992).

[40] P. Hayden and J. Preskill, J. High Energy Phys. 09 (2007) 120

[41] Y. Sekino and L. Susskind, J. High Energy Phys. 10 (2008) 065.

[42] P. M. Chesler and L. G. Yaffe, Phys. Rev. Lett. 102, 211601 (2009).

[43] M. P. Heller, R. A. Janik, and P. Witaszczyk, Phys. Rev. Lett. 108, 201602 (2012).

[44] P. Kovtun, D. T. Son, and A. O. Starinets, J. High Energy Phys. 10 (2003) 064. 